\title{
EFECTOS DE DOS TÉCNICAS DE MANEJO AGRONÓMICO DEL PEPINO (Cucumis sativus L.) SOBRE LA OCURRENCIA POBLACIONAL DE INSECTOS PLAGAS, BENÉFICOS Y EL RENDIMIENTO EN TISMA, MASAYA
}

\section{Edgardo Jiménez-Martínez ${ }^{1}$, Marjorie Padilla Mejía}

${ }^{1} \mathrm{Ph} . D$. Entomología, Universidad Nacional Agraria, Managua, Nicaragua. km 12 1/2 Carretera Norte

E-mail: edgardo.jimenez@una.edu.ni, Telef. 2233-1265,Fax: 2233-1267,2263-2609

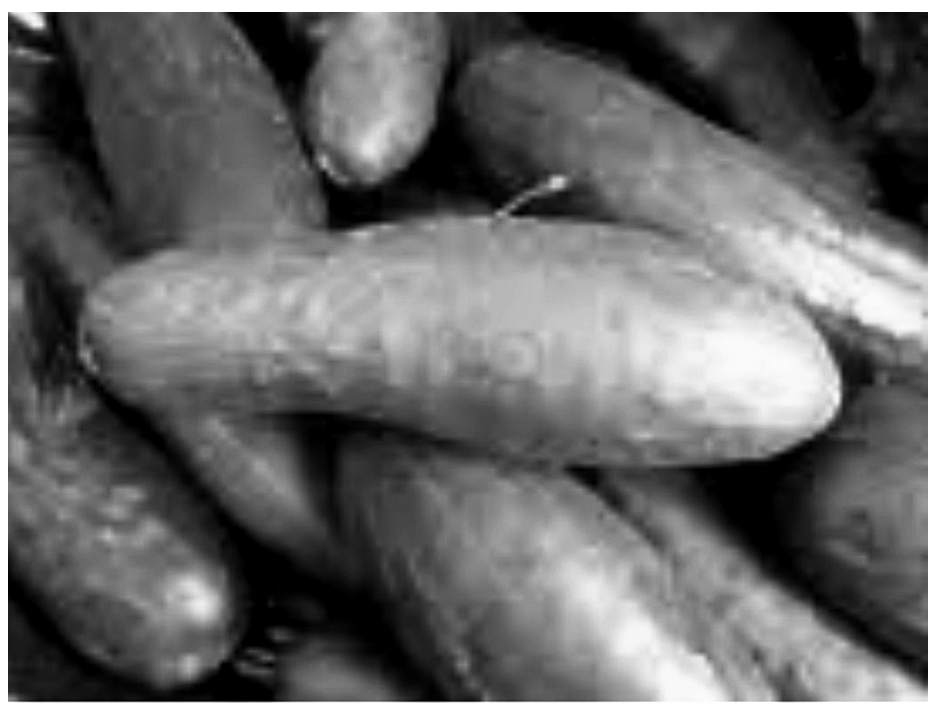

\section{RESUMEN}

Este estudio se realizó con el objetivo de comparar el efecto que tienen dos técnicas de manejo agronómico del cultivo del pepino, sobre los insectos plagas, insectos benéficos y el rendimiento. El estudio se estableció en la finca "Berlín" ubicada en el municipio de Tisma - Masaya, llevándose a cabo entre los meses de diciembre 2006 a febrero 2007. Para el desarrollo de esta investigación se establecieron dos parcelas, en una parcela el pepino se desarrolló de forma rastrera y en la otra en espaldera. En cada parcela se tomaron semanalmente las siguientes variables: número de insectos plagas y benéficos por planta, y durante la cosecha el rendimiento del cultivo, para comparar el rendimiento entre las dos técnicas de siembras y para realizar un análisis de presupuesto parcial. Los resultados obtenidos fueron los siguientes: el menor número de insectos plagas lo presentó la parcela de pepino rastrero comparado con pepino sembrado en espaldera. Encontrándose diferencias significativas entre ambas técnicas de siembras. También se observó una tendencia de mayor número de insectos benéficos en la parcela de pepino rastrero en comparación con pepino en espaldera, aunque no se encontró diferencias significativas entre ambas técnicas de siembra. El mejor rendimiento y mayor beneficio neto lo presentó la parcela de pepino en espaldera comparado con la parcela de pepino rastrero.

Palabras clave: Pepino, insectos plagas, insectos benéficos, rendimiento.

\begin{abstract}
This study was carried out with the objective to compare the effect of two cucumber management agronomic techniques on insect pest, benefitial insects and yield. The study was established on the farm "Berlin" located on the municipality of Tisma, Masaya, between the months of December 2006 and February 2007. To perform this study, two parcels were established, in one parcel, the cucumber was established on the soil floor technique as normal agronomic use in Tisma, and on the other parcel, the cucumber was established on a hanged wall post agronomic technique. On each parcel several variables were taken weekly as follow: Number of insect pest and benefic per plant, and during harvest, total yield was recorded in order to compare total yield between both agronomic techniques and to perform a partial budget economic analysis. Results were as follow, less number of insect pest were found on cucumber planted at soil level as compared with the hanged cucumber. Statistical differences were found between both growing techniques. In addition, a trend on higher number of beneficial insects was found on cucumber growth at soil level than posted cucumber, even though, no statistical differences were found among planting techniques. The best cucumber yield and the best net benefit were found on hanged cucumber compared to soil level cucumber.
\end{abstract}

Keywords: Cucumber, insect pest, beneficial insects, yield. 


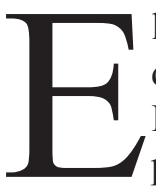

1 pepino (Cucumis sativus L.) es originario de las regiones húmedas y tropicales de la India, pero algunos autores consideran que primeramente llegó a china y posteriormente a otras regiones asiáticas, antes de ser llevado a Europa (Huerres, 1998). Este cultivo es considerado originario del sur de Asia, donde ha sido cultivado por más de 300 años, el pepino es una planta herbácea anual, pertenece al orden de las cucurbitales, y a la familia de las cucurbitácea. El pepino es una hortaliza fresca que cada día la consume más la población, y representa para el agricultor una alternativa para diversificar y satisfacer la demanda del mercado interno, en su contenido nutricional esta hortaliza contiene vitaminas A, B, C y minerales que son indispensables en la alimentación humana (CENTA, 2003).

El pepino es una planta anual, con tallo postrado, con un eje principal que da origen a ramas laterales de dimensiones variables $(100$ a $250 \mathrm{~cm})$, las plantas son altamente susceptibles a las bajas temperaturas, la floración se inicia con flores masculinas que se presentan en racimos, cuando la planta esta aún relativamente pequeña, las flores femeninas aparecen posteriormente y miden de 2-3 cm de diámetro y sus pétalos son de color amarillo, algunas veces aparecen flores perfectas y hermafroditas. En dependencia de las características biológicas, las variedades oscilan entre 45-75 días. Las principales variedades usadas en América Central vienen de Estados Unidos y se distinguen por la presencia o ausencia de espinitas blancas en los frutos, por el largo, el color de la fruta y por su característica de resistencia a enfermedades. En la actualidad el valor alimenticio del pepino contiene $95-96 \%$ de agua, pero su agradable sabor y el complemento que constituye para las comidas ricas en grasa, proteínas y alimentos calóricos hace que tenga una buena aceptación por la población (Avelares, 2001).

A nivel internacional México es el principal productor y exportador de pepino logrando producir 434792 toneladas, y logrando exportar 386094 toneladas en el año 2002 a países como: China, Turquía, Irán, Estados Unidos y Japón, posteriormente le siguen los países bajos, España, Jordania y otros, logrando una exportación de1 505905 toneladas en el 2002 (Ospina y Alonso, 1998).

En Nicaragua se estima que el área que se cultiva anualmente es de 315 a 365 hectáreas, localizándose, casi toda la mitad de la producción en el norte del país en los departamento de Jinotega y Matagalpa con rendimientos promedios de 10 toneladas por hectáreas. Otras regiones donde se cultiva en pequeñas escalas son los departamentos de: Masaya, Granada y Rivas. En el departamento de Masaya el pepino se siembra en mayores cantidades en el municipio de Tisma, sobre todo en la época de verano donde el área cultivada se estima en unas 20 hectáreas anualmente (MIDINRA, 1984).

Según Cáceres, (en 1980) se reconocieron dos tipos de pepino, un primer tipo para uso de fresco, ensaladas y un segundo tipo para el uso de encurtidos. Algunos productores de pepino dicen que son aromáticos y muy adecuados para la utilización de postres.

El pepino es una de las hortalizas que cada día más consume nuestra población debido a que contiene un gran valor nutricional en cuanto a vitaminas y minerales (CENTA, 2003).

Los problemas fitosanitarios que se presentan en este cultivo, provienen generalmente de los alrededores y de plantas de la misma familia botánica. Es importante mencionar que el pepino esta expuesto a una gran cantidad de limitaciones que afectan su desarrollo, reduciendo su rendimiento, y desmejorando la calidad de los frutos, entre estos factores que limitan su desarrollo están los de origen biótico, como plagas (mosca blanca, áfidos, barrenador de los brotes, nematodos y tortuguillas) y enfermedades como: Oidium o mildium polvoriento, mancha angular, mosaico, y antracnosis (Montes, 2005).

El cultivo del pepino es una planta herbácea, anual sus guías se pueden extender libremente sobre el suelo, como también puede trepar ayudada por sus zarcillos. Comúnmente el pepino se cultiva sobre el suelo en diferentes épocas de siembra, esto se debe por el desconocimiento de técnicas adecuadas de manejo en la mayoría de los casos y en otros por el costo adicional que significa una estructura para sostenerlo (CENTA, 2003).

Hasta el día de hoy son muchos los conocimientos que tenemos, pero cada día se pretende conocer más sobre cultivos hortícola, en este caso en el cultivo del pepino existen formas estratégicas de siembra como son en espaldera, que es una técnica no muy usada pero vieja de existir, esta técnica agronómica de sembrar el pepino se realiza con el propósito de obtener productos de mejor calidad. La técnica agronómica de tutores ó espaldera se utiliza para conducir las plantas ya que esta posee hábito trepador y crecimiento indeterminado, con el uso de esta técnica se obtiene mayores beneficios ya que la calidad de los frutos mejora cuando el pepino se produce en espaldera, los beneficios que se obtienen con esta técnica son: frutos más uniformes, mejor aprovechamiento de energía solar, menor influencia 
de enfermedades, mejor color de los frutos y mayores poblaciones de plantas por área y facilita la cosecha, lo contrario de la técnica rastrera (Castillo, 1970).

Las actividades agropecuarias en el municipio de Tisma ocupan el primer lugar en la economía, la cual genera un $77 \%$ del total de empleos existentes; en la agricultura predominan los cultivos de hortalizas (AMUNIC, 2005).

Actualmente en Tisma el cultivo de hortalizas esta en manos de pequeños productores, sin embargo uno de los problemas fundamentales que presentan dichos productores, son los bajos rendimientos que alcanzan por unidad de superficie y la mala calidad de los frutos que hacen que disminuyan su valor comercial; lo que incide en la economía del país, esta problemática se genera debido a que comúnmente los productores de esta zona, realizan siembras inadecuadas de los cultivos como por ejemplo las siembras de pepino rastrero, lo que ha contribuido a incrementar los problemas fitosanitarios provocados sobre todo por plagas como: mosca blanca, pulgones e insectos chupadores como chinches.

Debido a esta problemática se realizó un estudio con el propósito de comparar el efecto de dos técnicas agronómicas en el cultivo del pepino una rastrera y otra en espaldera y conocer su efecto sobre la ocurrencia poblacional de los insectos plagas, benéficos, y el rendimiento.

\section{MATERIALES Y MÉTODOS}

Ubicación del estudio. El estudio se realizó en la finca "Berlín" propiedad del productor Francisco Javier Altamirano donde se establecieron 2 parcelas de pepino una de forma rastrera y la otra en espaldera.

Descripción del municipio de Tisma. El estudio se realizó en el municipio de Tisma departamento de Masaya, ubicado en la parte noroeste de la capital Managua, entre las coordenadas $12^{\circ} 04^{\prime}$ latitud norte y $86^{\circ} 01^{\prime}$ latitud oeste, posee una superficie de $124.98 \mathrm{~km}^{2}$, con una población 11063 habitantes donde el 71\% de la población es rural y el $29 \%$ de la población es urbana. El clima se caracteriza por ser tropical de sabana con $\mathrm{T}^{\mathrm{o}}$ promedios de $27.5 \mathrm{C}$ y con precipitaciones pluviales anuales que oscilan entre los 1200 y $1400 \mathrm{~mm}$ y se encuentra a $50 \mathrm{msnm}$.

La mayor parte de la población rural se encuentra en condiciones de pobreza y tienen un sistema de producción de autoconsumo con una alta incidencia de plagas que afectan los rendimientos de las hortalizas, la forma de manejo de las plagas se realiza principalmente con productos químicos (INEC, 1998; INIFOM, 2006).
Descripción de la parcela en estudio. Se seleccionó el municipio de Tisma, porque es una zona altamente productora de pepino y porque existe una gran problemática fitosanitaria provocada por insectos plagas. El estudio se realizó entre diciembre del 2006 y febrero 2007 y consistió en una comparación por parcelas sin repeticiones, donde se evaluaron dos técnicas de manejo agronómico del pepino entre dos parcelas, una parcela de pepino sembrado de forma rastrera y una de pepino sembrado en espaldera. Ambas parcelas constaban con un área de $32 \mathrm{~m}$ de largo x $30 \mathrm{~m}$ de ancho para un total de $960 \mathrm{~m}^{2}$ cada parcela.

\section{Manejo agronómico del cultivo.}

Material genético. La variedad de semilla de pepino que se utilizó en el experimento fue la variedad Dasher la más utilizada por los productores en esta zona, esta variedad híbrida completa su ciclo entre los 60 a 75 días de maduración y con un peso promedio de $0.23 \mathrm{~kg}$.

Preparación del terreno. El terreno se preparó realizando roza, quema, dos pase de romplona, también se realizó una pasada de arado a $35 \mathrm{~cm}$ de profundidad, 15 días antes del transplante, para aprovechar que todo el rastrojo que se incorporara al suelo tuviera suficiente tiempo para descomponerse.

Época de siembra. El trabajo se desarrollo en la época de apante o riego. Las distancias de siembra en este estudio fueron de 2.5 vrs entre plantas y 3 vrs entre surco, dejando 3-4 semillas por golpe enterradas a una profundidad de $2 \mathrm{~cm}$, a los 10-12 días después de germinada la planta cuando ya había formado la segunda hoja se efectúo un raleo, dejando las 2 plantas mejores desarrolladas.

Aporque. Se realizó de forma manual, y en una sola ocasión (18 días despues de siembra), el objetivo de esta práctica fue proporcionar aireación y mayor anclaje al sistema radicular. El momento más aconsejable para realizar esta actividad es después de la fertilización al suelo, pues ayuda a incorporar el fertilizante del mismo.

Riego. El agua de riego que se le suministro a las plantas se extrajo de un pozo haciendo uso de una moto-bomba de 2.5 pulgadas de salida, esta se llevaba hasta las zanjas de los surcos por medio tubos galvanizados. El riego se hacia 2 veces por semana durante los primeros 45 DDS y posteriormente se hizo un riego semanal. 
Entutorado y amarre. Los labores de tútoreo se realizó para proveer a la planta un soporte ó punto de apoyo a medida que avanzaba en su crecimiento, esto es muy importante en variedades o híbridos cuya altura supera los $1.2 \mathrm{~m}$ de altura, ya que la carga que producen es capaz de agobiar a la planta misma. Esta actividad se realizó el cinco de enero a los 25 DDS, para la construcción de este sistema se utilizó cabuyas y postes en cada hilera de tutores los que sirvieron para fijar la planta verticalmente.

Fertilización. La fertilización de las parcelas en estudio se realizó utilizando, productos tanto foliares como incorporados al suelo. La fertilización se efectúo a los seis y veintisiete días después de la siembra. Dentro de los foliares podemos mencionar: Boro, Calcio y Zinc. Entre los productos incorporados al suelo están: 15-30-15, 20-20-20, 11-6-44.

Manejo fitosanitario de plagas y enfermedades en etapa de campo. Para el manejo de plagas y enfermedades, se utilizaron insecticidas y fungicidas tanto químicos como biológicos: Engeo $\AA$, Tigre $\AA$, Nomolt $\AA$, Carbendazim $\AA$, Mancozeb $\AA$, Benomyl $\AA$, Agrimicin $\AA$, Neem $\AA$, y Javeling $\AA$, usados para controlar plagas como mosca blanca, gusano del fruto, entre otras plagas y enfermedades.

Cosecha. Al momento de la cosecha es importante determinar el tamaño del fruto. La cosecha se comenzó el 16 de Enero, realizándose un total de 14 cortes. Los cuales se realizaron durante las últimas horas de la tarde, sin dañar las guías, el pepino destinado a la comercialización se cosechaba cada dos o tres días, luego estos se clasificaron en categorías para cumplir con las exigencias del mercado. La cosecha se vendió en el mercado de Masaya y en ventas locales del municipio de Tisma.

\section{VARIABLES EVALUADAS}

Número, descripción e identificación de insectos plagas y benéficos. Para determinar las poblaciones de insectos plagas y benéficos en las diferentes parcelas en estudio, se realizaron recuentos semanales para los cuales se seleccionaron cinco puntos al azar por parcelas, de cada punto se tomaron 30 plantas para un total de 150 plantas muestreadas por tratamiento, en cada una de las treinta planta por punto se tomaba la guía principal donde se revisaban las hojas por el haz y el envés, se muestreaban los brotes terminales, flores y frutos para determinar la presencia de insectos plagas y benéficos.
La toma de datos en cada parcela (rastrera y espaldera), se realizó en horas de la mañana y semanalmente entre los meses de diciembre 2006 a febrero 2007.

Para la identificación y clasificación de los insectos se uso un estereoscopio marca (VAN GUARD-1264SF, SN 009028) cuya lente ocular es de 10 x de resolución. Donde se examinaban las principales características morfológicas de las familias de los insectos, igualmente para la identificación se hizo uso de referencias de bibliográficas (libros) así como también se realizaron comparaciones de nuestros especimenes con especimenes de insectos de referencias del museo entomológico de la UNA

Rendimiento. Para obtener los datos de rendimiento se realizaron cosechas de acuerdo a lo que producían las parcelas. Se seleccionaron 10 plantas por tratamiento para un total de 20 plantas en ambas parcelas, se recolectaron los frutos, seguidamente se pesó el total de frutos por plantas para obtener el peso en gramos y posteriormente convertirlo a $\mathrm{kg} \mathrm{ha}^{-1}$.

Análisis de presupuesto parcial. Se comenzó por recolectar los costos que varían (mano de obra para las aplicaciones de control químico y control biológico) de un tratamiento a otro con el fin de obtener los costos y beneficios de las técnicas utilizadas, luego se calculó el rendimiento y el precio unitario para luego calcular los beneficios brutos, a esto se le resto los costos variables para obtener el Beneficio neto.

Análisis estadístico de los datos. A cada una de las variables registradas en el experimento se les realizó un análisis de varianza (ANDEVA, PROC, GLM en SAS), y a los ANDEVA que resultaron con probabilidades significativas $(P \leq$ de 0.05$)$ se les realizó una prueba de separación de medias por medio de la prueba de $t$ de student (SAS instituto, 1990).

\section{RESULTADOS}

Los principales insectos plagas y benéficos encontrados en este estudio en el cultivo del pepino, se presentan en el Tabla 1. Los insectos encontrados en pepino rastrero y pepino en espaldera fueron mosca blanca (Bemicia tabaci Gennnadius.), Gusano verde (Diaphania hyalinata L.), Áfidos (Aphis gossypii Glover.), Chinche rápida (Creontiades rubrinervis Stal), Crisomélidos (Diabrotica sp), Se encontraron algunos insectos benéficos en ambas parcelas tales como: Hormigas, abejas y Arañas. 
Tabla 1. Insectos plagas y benéficos encontrados en el cultivo del pepino entre los meses de Diciembre, 2006, a Febrero 2007 en Tisma, Masaya.

\begin{tabular}{llllll}
\hline \multicolumn{5}{c}{ Insectos plagas } \\
\hline N. Común & Orden & Familia & Género & sp & Categoría \\
\hline Gusano verde & Lepidoptera & Pyralidae & Diaphania & hyalinata & Masticador \\
Áfidos & Hemiptera & Aphididae & Aphis & gossypii & Chupador \\
Mosca blanca & Hemiptera & Aleyrodidae & Bemisia & tabaci & Chupador \\
Crisomélidos & Coleoptera & Crisomelidae & Diabrótica & sp. & Masticador \\
Chinche rápida & Hemiptera & Miridae & Creontiades & rubrinervis & Chupador \\
\hline \multicolumn{7}{|c}{} & \multicolumn{2}{c}{ Insectos benéficos } & & \\
\hline Arañas & - & Aracnidae & - & - & Depredador \\
Hojas & Himenóptera & Apidae & Apis & mellifera & Polinizador \\
\hline & Himenóptera & Formicidae & Solenopsis & sp. & Depredador \\
\hline
\end{tabular}

Comparación del promedio total de insectos plagas y benéficos. Se comparó el promedio total de insectos plagas y benéficos encontrados en la parcela de pepino rastrero y pepino en espaldera (Figura 1). El promedio total de insectos plagas encontrados en la parcela de pepino en espalderas fue mayor comparado con el número de total de insectos plagas encontrados en la parcela de pepino rastrero, en cambio el número total de insectos benéficos encontrados en la parcela de pepino rastrero fue mayor comparado con el número total de insectos benéficos encontrados en la parcela de pepino en espaldera. Al realizar el análisis de varianza para comparar la ocurrencia poblacional de insectos plagas y benéficos en ambos sistemas de siembra. Se encontró diferencias significativas en la poblaciones de insectos plagas con probabilidad de $P=0.001$, presentando la menor población de insectos plagas pepino rastrero con 11.62 insectos por planta comparado con pepino en espaldera que presento 19.17 insectos por planta. Sin embargo no se encontró diferencias significativas en la ocurrencia poblacional de insectos benéficos entre ambos tratamientos (Tabla 2).

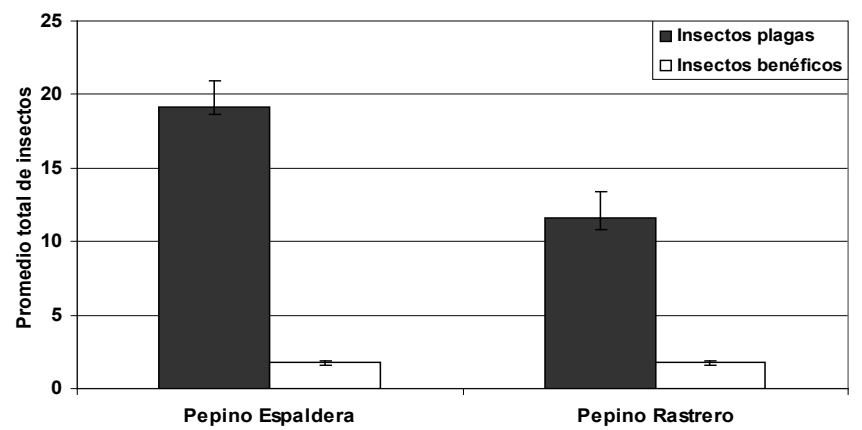

Figura 1. Promedio total de insectos encontrados en el cultivo de pepino rastrero y espaldera, en Tisma, Masaya, Diciembre 2006 y Febrero 2007.
Tabla 2. Análisis de varianza de la ocurrencia poblacional del promedio total de insectos plagas y benéficos (Media \pm ES) encontrados en pepino rastrero, versus pepino en espaldera, en Tisma, Masaya, Diciembre 2006 - Febrero 2007.

\begin{tabular}{lll}
\hline Tratamientos & Plagas & Benéficos \\
\hline Pepino rastrero & $11.62 \pm 0.47 \mathrm{a}$ & $1.73 \pm 0.17$ \\
Pepino en espaldera & $19.17 \pm 0.85 \mathrm{~b}$ & $1.76 \pm 0.13$ \\
CV & 119.06 & 63.00 \\
$P$ & $0.001(\mathrm{DS})$ & $0.91(\mathrm{NS})$ \\
$\mathrm{F} ; d f ; \mathrm{n}$ & $65.07 ; 1478 ; 670$ & $0.01 ; 103 ; 67$
\end{tabular}

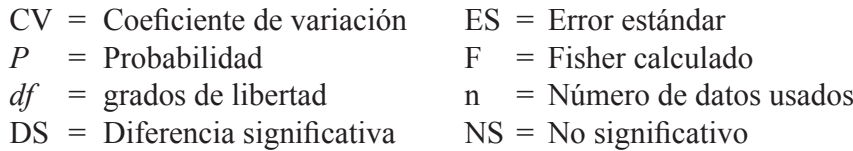

Ocurrencia poblacional de gusano verde (Diaphania hyalinata). Se comparó la ocurrencia poblacional de gusano verde en el cultivo del pepino rastrero y espaldera (Figura 2). En la parcela de pepino rastrero se observó que las poblaciones del gusano verde se presentaron desde la primera fecha de muestreo 27 de diciembre ocurriendo su mayor pico poblacional con 3.3 gusanos por planta, en la fecha 10 de enero, luego estas poblaciones descendieron hasta llegar a la fecha 9 febrero donde las poblaciones de larvas de D. hyalinata, fue nula.

En el pepino en espaldera las poblaciones de gusano verde se presentaron a partir del 27 de diciembre, ocurriendo su mayor pico poblacional en la fecha 3 de enero con 3.8 gusanos por planta. Estas poblaciones descendieron hasta la fecha 9 de febrero. Al realizar el análisis de varianza para comparar la ocurrencia 
poblacional de gusanos verde, en ambos sistemas de siembra, no se encontró diferencias significativas $(\mathrm{P}=$ 0.526 ) Las medias de tratamientos (Media $\pm \mathrm{ES}$ ), fueron $4.20 \pm 0.12$ para pepino rastrero y $4.09 \pm 0.12$ para pepino en espaldera (Tabla 3 ).

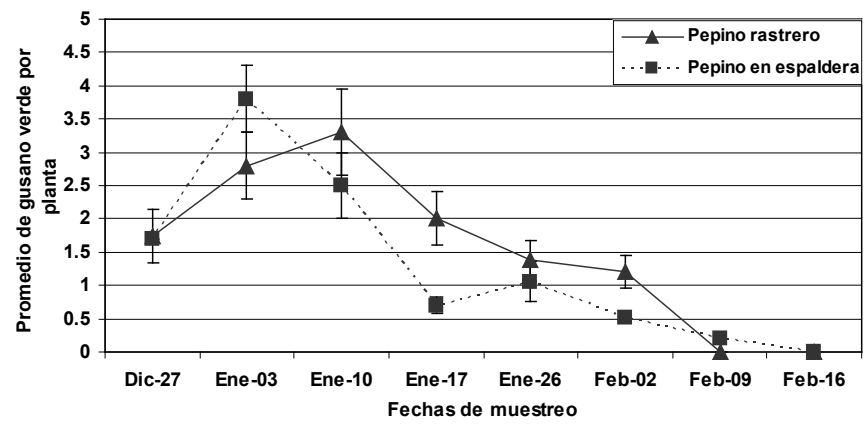

Figura 2. Ocurrencia poblacional de gusano verde (Diaphania hyalinata) en el Cultivo del pepino rastrero y pepino en espaldera en Tisma, Masaya entre Diciembre 2006 y Febrero 2007.

Tabla 3. Análisis de varianza de la ocurrencia poblacional del gusano verde Diaphania hyalinata (Media \pm ES) y Aphis gossypii (Media $\pm \mathrm{ES}$ ) encontrados en pepino rastrero versus pepino en espaldera, en Tisma, Masaya, Diciembre 2006- Febrero 2007

\begin{tabular}{lll}
\hline Tratamientos & Diaphania hyalinata & Aphis gossypii \\
\hline Pepino rastrero & $4.20 \pm 0.12$ & $16.15 \pm 0.77 \mathrm{a}$ \\
Pepino en espaldera & $4.09 \pm 0.12$ & $34.04 \pm 1.25 \mathrm{~b}$ \\
CV & 62.09 & 81.82 \\
$P$ & $0.526(\mathrm{NS})$ & $0.0001(\mathrm{DS})$ \\
$\mathrm{F} ; d f ; \mathrm{n}$ & $0.40 ; 831 ; 387$ & $162 ; 35 ; 333$ \\
\hline
\end{tabular}

$\mathrm{CV}=$ Coeficiente de variación

$P \quad=$ Probabilidad

$\mathrm{ES}=$ Error estándar

$d f=$ grados de libertad

$\mathrm{F}=$ Fisher calculada

$\mathrm{n}=$ Número de datos usados

NS $=$ No significativo

Ocurrencia poblacional de áfidos Aphis gossypii. Se comparó la ocurrencia poblacional de las colonias de áfidos en el cultivo del pepino rastrero y pepino en espaldera (Figura 3). Se observó que las poblaciones de colonias de áfidos se presentaron desde la primera fecha de muestreo 27 de diciembre en ambos sistemas de siembra. En la parcela de pepino rastrero, las poblaciones de colonias de áfidos presentaron su mayor pico poblacional en la fecha 16 de febrero con 20.5 colonias de áfidos por planta y en la parcela de pepino en espaldera el mayor pico poblacional se presentó en la fecha 09 de febrero con 35 colonias de áfidos por planta. Al realizar el análisis de varianza para comparar la ocurrencia poblacional de áfidos, en ambos sistemas de siembra se encontró diferencias significativas con probabilidad de $(P=0.0001)$ y al realizar la separación de medias con la prueba t de student, se encontró que el menor número de áfidos lo presentó la parcela de pepino rastrero con un promedio de $16.15 \pm 0.77$ colonias de áfidos por planta, comparado con la parcela de pepino en espaldera que presentó un promedio de $34.04 \pm 1.25$ colonias de áfidos por planta (Tabla 3 ).

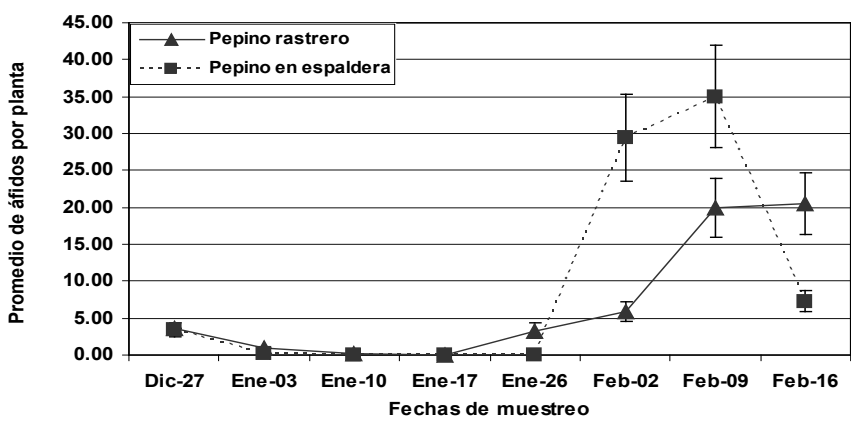

Figura 3. Ocurrencia poblacional de áfidos (Aphis gossypii) en el cultivo del pepino rastrero y pepino en espaldera, en Tisma, Masaya entre Diciembre 2006 y Febrero 20007.

Ocurrencia poblacional de mosca blanca. Se comparó la ocurrencia poblacional de mosca blanca en los sistemas de pepino en el suelo y pepino en espaldera (Figura 4). En la parcela de pepino rastrero se observó que las poblaciones de mosca blanca se presentaron a partir de la segunda fecha de muestreo 3 de enero, ocurriendo su mayor pico poblacional en la fecha 2 de febrero con 1.6 mosca blanca por planta y en la parcela de pepino en espaldera las poblaciones de mosca blanca se presentaron desde la primera fecha de muestreo 27 de diciembre, ocurriendo su mayor pico poblacional en la fecha 26 de enero con 1.8 mosca blanca por planta. Al realizar el análisis de varianza para comparar la ocurrencia poblacional de mosca en ambos sistemas de siembra, no se encontró diferencias significativas (Tabla 4).

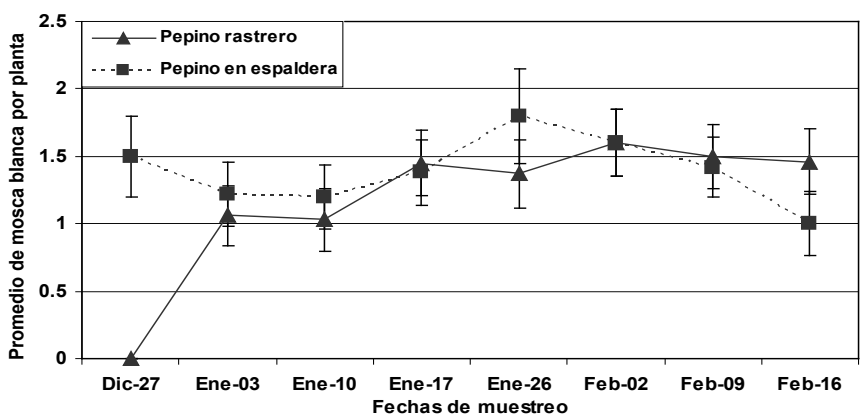

Figura 4. Ocurrencia poblacional de Bemissia tabaci en los sistemas de pepino rastrero y pepino en espaldera en Tisma, Masaya entre Diciembre 2006 y Febrero 2007. 
Ocurrencia poblacional del chinche rápida (Creontiades rubrinervis). Se comparó la ocurrencia poblacional de chinche rápida en el sistema de siembra de pepino rastrera y pepino en espaldera (Figura 5). En la parcela de pepino rastrero las poblaciones de chinche se presentaron desde la primera fecha de muestreo 27 de diciembre y ocurriendo su mayor concentración en esa misma fecha con 0.2 chinche rápida por planta. En la parcelas de pepino en espaldera las poblaciones de este insecto solamente se observaron en las fechas 17 y 26 con un promedio de 0.04 chinches por planta. Al realizar el análisis de varianza para comparar la población de chinches rápida, en ambos sistemas de siembra, no se encontró diferencias significativas (Tabla 4).

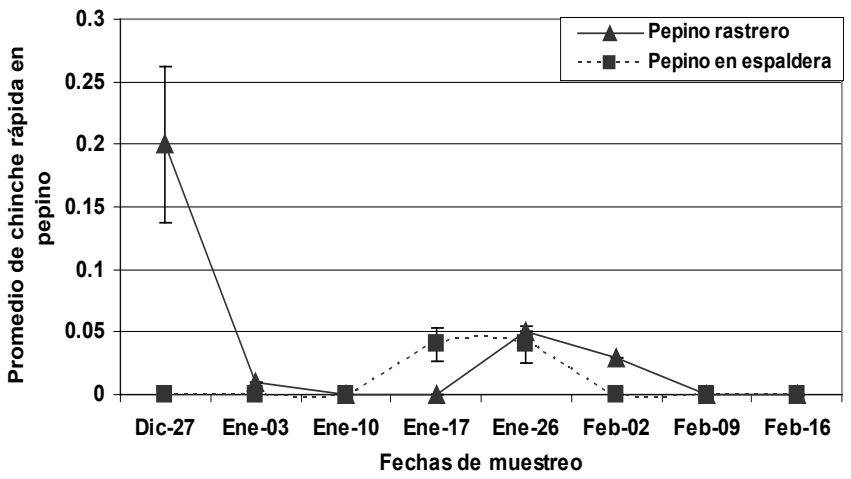

Figura 5. Ocurrencia poblacional de chinche rápida (Creontiades rubrinervis) en el cultivo de pepino rastrero y de pepino en espaldera en Tisma, Masaya entre Diciembre 2006 y Febrero 2007.

Tabla 4. Análisis de varianza de la ocurrencia poblacional de Bemisia tabaci y Creontiades rubrinervis (Media \pm ES) encontrados en pepino rastrero versus pepino en espaldera, en Tisma, Masaya, Diciembre 2006- Febrero 2007

\begin{tabular}{lll}
\hline Tratamientos & Bemisia tabaci & $\begin{array}{l}\text { Creontiades } \\
\text { rubrinervis }\end{array}$ \\
\hline Pepino rastrero & $1.35 \pm 0.08 \mathrm{a}$ & $2.78 \pm 0.39$ \\
Pepino en espaldera & $1.46 \pm 0.08 \mathrm{a}$ & $1.33 \pm 0.23$ \\
CV & 67.04 & 89.32 \\
$P$ & $0.3514(\mathrm{NS})$ & $0.086(\mathrm{NS})$ \\
$\mathrm{F} ; d f ; n$ & $0.87 ; 259 ; 261$ & $3.07 ; 45 ; 38$ \\
\hline
\end{tabular}

$\mathrm{CV}=$ Coeficiente de variación

$P=$ Probabilidad

$d f=$ grados de libertad

$\mathrm{ES}=$ Error estándar

$\mathrm{F}=$ Fisher calculada

$\mathrm{DS}=$ Diferencia significativa
Ocurrencia poblacional de crisomélidos Diabrótica $\boldsymbol{s p}$. Se comparó la incidencia poblacional de crisomélidos en los sistemas de siembra de pepino rastrero y pepino en espaldera (Figura 6). Las poblaciones de crisomélidos en ambos sistemas de siembra se observaron a partir de la primera fecha de muestreo 27 de diciembre ocurriendo su mayor pico poblacional en esa misma fecha, después las poblaciones de pepino rastrero descendieron hasta la fecha 17 de febrero, encontrándose en la fecha posterior 26 de enero 0.01 crisomélidos por planta. En cambio en pepino en espaldera su población descendió el 3 de enero observando un pequeño incremento en la fecha 10 de enero posteriormente su población descendió hasta ser nula en la fecha 26 de febrero. Al realizar el análisis de varianza para comparar la ocurrencia poblacional de crisomélidos en ambos sistemas de siembra, no se encontró diferencias significativas (Tabla 5).

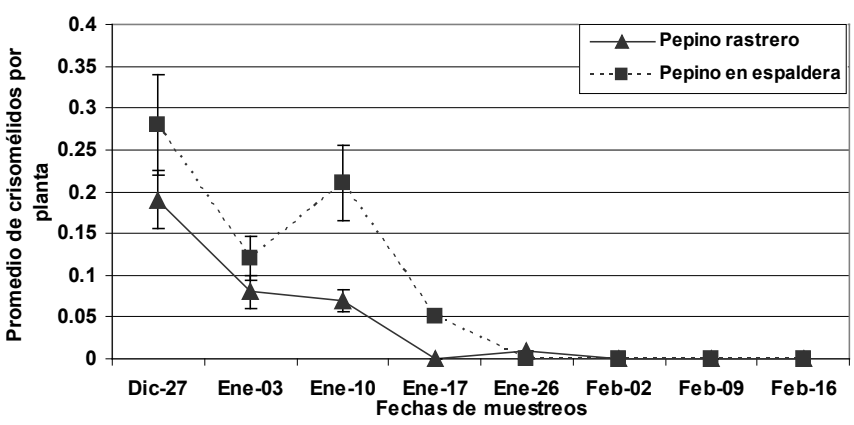

Figura 6. Ocurrencia poblacional de crisomélidos Diabrotica $s p$. en los sistemas de cultivo de pepino rastrero y pepino en espaldera en Tisma, Masaya entre Diciembre 2006 y Febrero 2007.

Ocurrencia poblacional de arañas. Se comparó la ocurrencia poblacional de arañas. En los sistemas de pepino rastrero y pepino en espaldera (Figura 7). En ambos sistemas de cultivos las poblaciones de arañas se presentaron a partir de la primera fecha de muestreo 27 diciembre. En la parcela de pepino rastrero el mayor pico poblacional se presento en la fecha 26 de enero con 0.4 arañas por planta, en las fechas posteriores sus poblaciones fueron nulas. En cambio en el sistema de pepino en espaldera las poblaciones de arañas fueron constantes hasta la fecha 26 de enero, presentando su mayor pico poblacional en la primera fecha de muestreo 27 de diciembre con 0.05 arañas por planta. Al realizar el análisis de varianza para comparar la incidencia poblacional de arañas, en ambos sistemas de siembra, no se encontró diferencias significativas entre ambos sistemas de siembra (Tabla 5). 


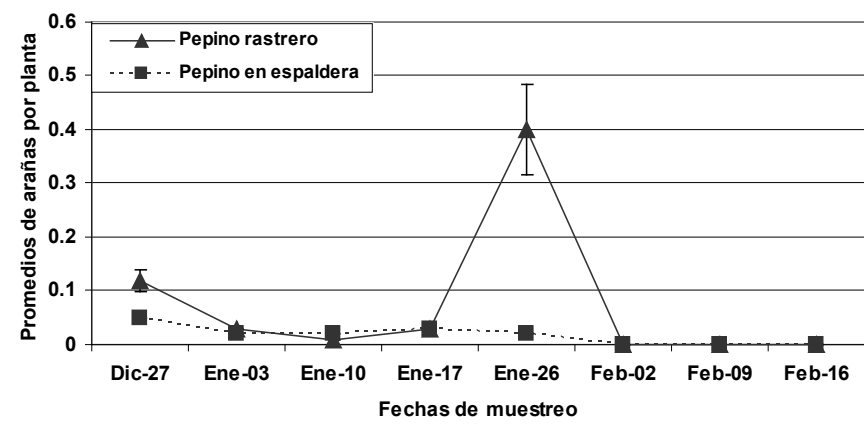

Figura 7. Ocurrencia poblacional de arañas en los sistemas de pepino rastrero y pepino en espaldera en Tisma, Masaya entre Diciembre 2006 y Febrero 2007.

Tabla 5. Análisis de varianza de la ocurrencia poblacional de Diabrótica $s p$ (Media \pm ES) y la ocurrencia poblacional de Arañas (Media \pm ES) encontrados en pepino rastrero versus pepino en espaldera en Tisma, Masaya, Diciembre 2006Febrero 2007

\begin{tabular}{lll}
\hline Tratamientos & Diabrótica sp & Arañas \\
\hline Pepino rastrero & $1.36 \pm 0.12$ & $5.83 \pm 1.87$ \\
Pepino en espaldera & $1.46 \pm 0.12$ & $1.00 \pm 0.38$ \\
CV & 65.76 & 119.130 \\
$P$ & $0.60(\mathrm{NS})$ & $0.490(\mathrm{NS})$ \\
F;df;n & $0.27 ; 101 ; 36$ & $0.51 ; 11 ; 12$ \\
\hline
\end{tabular}

$\mathrm{CV}=$ Coeficiente de variación

$P=$ Probabilidad

$\mathrm{ES}=$ Error estándar

$d f=$ grados de libertad

$\mathrm{F}=$ Fisher calculada

NS $=$ No Significativo

Ocurrencia poblacional de abejas. Se comparó la ocurrencia poblacional de abejas en los sistemas de pepino rastrero y pepino en espaldera (Figura 8). Las poblaciones de abejas se presentaron a partir de la primera fecha de muestreo 27 de diciembre en ambos sistemas de siembra con un promedio de 3.4 abejas por planta, después sus poblaciones descendieron, posteriormente se observó en la parcela de pepino rastrero que sus poblaciones incrementaron a partir del 26 de febrero alcanzando su mayor pico poblacional en la fecha 16 de febrero con 20.5 abejas por planta en cambio en pepino en espaldera las poblaciones de abejas aumentaron a partir de la fecha 2 de febrero, alcanzando su mayor pico poblacional el 9 de febrero con 35 abejas pos planta, posteriormente su población presento un descenso. No se realizó análisis estadístico de esta variable, pero desde la fecha 26 de enero a la fecha febrero 16 se notó mayor número de estos insectos en las parcelas de pepino rastrero que en espaldera.

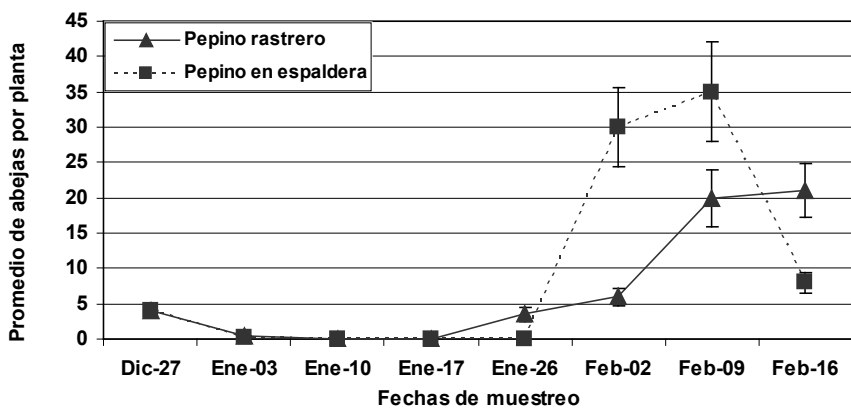

Figura 8. Ocurrencia poblacional de abejas en los sistemas de pepino rastrero y pepino en espaldera en Tisma Masaya entre Diciembre 2006 y Febrero 2007.

Ocurrencia poblacional de hormigas. Se comparó la ocurrencia poblacional de hormigas en los sistemas de siembra de pepino rastrero y pepino en espaldera (Figura 9). La ocurrencia poblacional de hormigas se presento desde la primera fecha 27 de diciembre en ambos sistemas de siembra. En pepino rastrero su mayor pico poblacional se presentó en la fecha 26 de enero con 0.1 hormiga por planta y en pepino sembrado en espaldera también su mayor pico lo presento en la fecha 26 de enero con 0.2 hormigas por planta. Después se observó que las poblaciones de hormigas en ambos sistemas de siembras descendieron en la fecha 2 de febrero. Al realizar el análisis de varianza para comparar la población de hormigas en ambos sistemas de siembra, se encontró diferencias significativas de con una probabilidad de $P=0.01$, y al realizar la separación de medias con la prueba $t$ de student, el mayor número de hormigas lo presentó la parcela de pepino rastrero con promedios de 2.40 hormigas por planta comparado con la parcela de pepino en espaldera que presentó un promedio 1.40 hormigas por planta (Tabla 6).

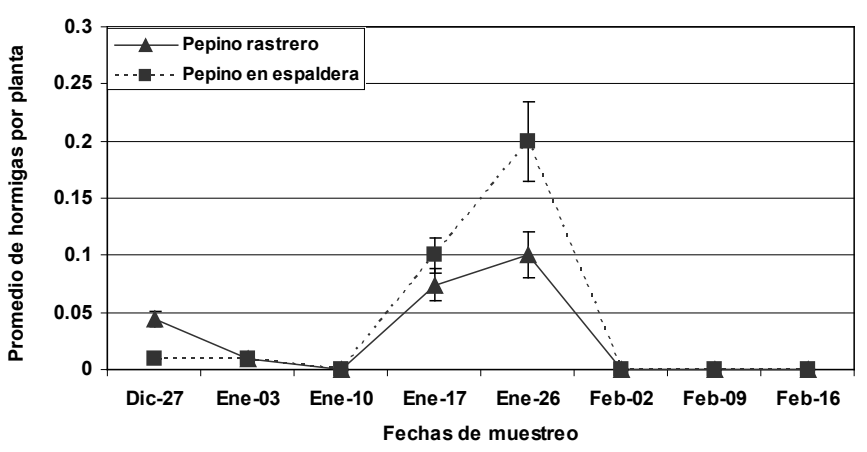

Figura 9. Ocurrencia poblacional de hormigas en los sistemas de pepino rastrero y pepino en espaldera en Tisma, Masaya entre Diciembre 2006 y Febrero 2007. 
Tabla 6. Análisis de varianza de la ocurrencia poblacional de Solenopsis sp. (Media \pm ES) encontrado en pepino rastrero versus pepino en espaldera en Tisma, Masaya, Diciembre 2006 y Febrero 2007

\begin{tabular}{ll}
\hline Tratamientos & Solenopsis sp. \\
\hline Pepino rastrero & $2.40 \pm 0.24 \mathrm{a}$ \\
Pepino en espaldera & $1.40 \pm 0.19 \mathrm{~b}$ \\
C.V & 42.37 \\
P & $0.01(\mathrm{DS})$ \\
F; $d f ; \mathrm{n}$ & $7.67 ; 18 ; 15$ \\
\hline
\end{tabular}

$\mathrm{CV}=$ Coeficiente de variación

$P=$ Probabilidad

$\mathrm{ES}=$ Error estándar

$d f=$ grados de libertad

$\mathrm{F}=$ Fisher calculado

DS = Diferencia significativa

El mayor rendimiento se obtuvo en el sistema de siembra pepino en espaldera (13 $045.31 \mathrm{~kg} \mathrm{ha}^{-1}$ ) comparado con el sistema de siembra de pepino rastrero que presento menor rendimiento por unidad de área $9061.04 \mathrm{~kg} \mathrm{ha}^{-1}$. Estos rendimientos equivalen a 39395.83 docenas de pepino por hectárea para pepino rastrero y 56718.75 docenas de pepino por hectárea para Pepino en espaldera.

Se realizó un análisis económico basado en un presupuesto parcial (Tabla 7), a través del cual se pudo determinar que el tratamiento pepino en espaldera obtuvo mayor beneficio neto con US \$ 2502 dólares comparado con pepino rastrero que presentó un beneficio neto de US \$ 1736 dólares.

Tabla 7. Presupuesto parcial en US \$ para cada tratamiento, diciembre 2006

\begin{tabular}{|c|c|c|}
\hline Rubro & $\begin{array}{l}\text { Pepino } \\
\text { rastrero }\end{array}$ & $\begin{array}{l}\text { Pepino en } \\
\text { espaldera }\end{array}$ \\
\hline Rendimiento (kg ha-1) & 9061 & 13045 \\
\hline Rendimiento ajustado (10\%) & 8155 & 11741 \\
\hline $\begin{array}{l}\text { Precio de campo por } \mathrm{kg} \text {. } \\
\text { de pepino en US } \$\end{array}$ & 0.2415 & 0.2415 \\
\hline Ingreso bruto/ha US \$ & 1969 & 2835 \\
\hline Costos & variables & US \$/ha \\
\hline Control químico & 133 & 133 \\
\hline $\begin{array}{l}\text { Control, biológico } \\
\text { CV }\end{array}$ & - & 50.82 \\
\hline Costos de las aplicaciones & 100 & 150 \\
\hline TOTAL CV & 233 & 334 \\
\hline Beneficio Neto/ha & 1736 & 2502 \\
\hline
\end{tabular}

Precio de venta por kg de pepino: 0.2415 \$ $/ \mathrm{kg}$

Cambio oficial de dólar $=$ C\$ 18.00

$\mathrm{CV}=$ Costos Variables

\section{DISCUSIÓN}

Según los resultados obtenidos en este estudio, el número total de insectos plagas encontradas en la parcela de pepino en espaldera fue mayor que el número total de insectos plagas encontrados en la parcela de pepino rastrero. En el análisis realizado se encontró diferencias significativas entre ambos sistemas de siembra. Según Nicholl et al., (1999) la razón de encontrar mayor número de insectos plagas en los cultivos solos se puede atribuir a que los monocultivos tienen alta concentración de biomasa disponible con facilidad para los fitófagos indeseables. También se observó que el número total de insectos benéficos fue mayor en la parcela de pepino rastrero comparado con la parcela de pepino en espaldera. Aunque no se encontró diferencias significativas entre ambos sistemas de siembra. Según Jiménez-Martínez (2008) las plantas producen olores como un mecanismo de atracción de insectos que le serán útiles especialmente a los polinizadores.

En este estudio los principales insectos plagas encontrados en las parcelas de pepino rastrero y pepino en espaldera fueron: Mosca blanca (Bemisia tabaci), áfidos (Aphis gossypii), gusano verde (Diaphania hyalinata L.), chinche rápida (Creontiades rubrinervis Stal.), crisomélidos (Diabrotica sp), de todos los insectos plagas encontrados los más importantes por los daños que ocasionan al cultivo del pepino son: mosca blanca (Bemisia tabaci), áfidos ( Aphis gossypii) y gusano verde (Diaphania hyalinata L.), la mosca blanca y los áfidos al alimentarse de la savia ocasionan daños directos e indirectos al debilitar la planta y transmitir virus como geminivirus y virus del tipo no persistente como el CMV (virus del mosaico del pepino), asimismo en la excreta se desarrolla el hogo comúnmente conocido como fumagina (Capnodium spp) el cual interfiere en la acción fotosintética de la planta, también deprecia el valor estético del fruto. El gusano verde se alimenta del follaje principalmente de las yemas terminales y durante la fructificación daña los frutos provocándoles perforaciones. Según Rosset, (1988), los herbívoros utilizan diferentes señales o indicadores para localizar a sus plantas hospederas e indica que si la señal es correcta la planta será atractiva y si la señal es incorrecta podría darse un efecto repelente para el insecto.

En la parcela de pepino en espaldera, se encontró el menor número de gusanos verde por planta comparado con pepino rastrero que presento una mayor ocurrencia. Aunque no se encontró diferencias significativas. Coincidiendo con García y Ángulo (2008) quienes encontraron las mayores poblaciones de gusanos verde en las parcelas de pepino sembrado solo. 
Al comparar la ocurrencia poblacional de áfidos en ambos sistemas de siembra, se encontró que la menor población de colonias de áfidos se presentó en la parcela de pepino rastrero. Al realizar el análisis de varianza de la ocurrencia poblacional de colonias de áfidos se encontró diferencia significativa. Las mayores poblaciones de áfidos se observaron en las últimas fechas de muestreo. Estos resultados coinciden con Pérez \& Sánchez, (2006) y García \& Ángulo, (2008) quienes encontraron la mayor ocurrencia de colonias de áfidos en las últimas fechas de muestreo. Según Argüello et al., (2007 B) los áfidos bajo condiciones de altas temperaturas se reproducen rápidamente, siendo su ciclo de vida de seis días, lo que explica su rápida explosión poblacional bajo condiciones de verano.

En el presente estudio se observa que las poblaciones de mosca blanca fueron mayores en pepino en espaldera que en pepino rastrero. Auque no se encontró diferencias significativas entre ambos sistemas de siembras. Según Hilje, (1993) las altas o bajas poblaciones de mosca blanca están influenciadas por condiciones ambientales como temperaturas, humedad relativa y precipitación. También se observo que la ocurrencia de chinches rápida fue menor en pepino espaldera que en pepino rastrero. Aunque al realizarle el análisis de varianza a la ocurrencia poblacional de chinches no se encontró diferencia significativas. Estos resultados son relativamente bajos comparados con los que obtuvieron García y Angulo, (2008) quienes encontraron altas poblaciones de chiches rápida.

Las poblaciones de crisomélidos en este estudio solamente se encontraron en las primeras fechas de muestreo, presentándose las menores poblaciones en la parcela de pepino rastrero comparado con pepino en espaldera. No encontrándose diferencias significas entre ambos sistemas de siembra.

En este estudio los principales insectos benéficos encontrados fueron: arañas, abejas (Apis mellifera) y hormigas (Solenopsis $s p$ ). Observándose que las arañas estuvieron presentes en casi todas las fechas de muestreo en los dos sistemas de siembra pepino rastrero y pepino en espaldera, en el caso de las abejas se observó mayor ocurrencia durante la etapa de floración y fructificación en pepino rastrero y pepino en espaldera, según JiménezMartínez, (2008) el olor o aroma de las flores juega un papel principal como atrayente de insectos polinizadores en las plantas angiospermas. Las abejas responden especialmente al aroma de las flores. La ocurrencia poblacional de las hormigas fue baja durante el estudio encontrándose mayor ocurrencia en las fechas 17 y 26 de enero. Según Altieri et al., (2007) la abundancia y diversidad de insectos benéficos dentro de un campo depende de la variedad de plantas en la vegetación que lo rodea, por lo que es importante establecer plantas que sean capaces de atraer enemigos naturales.

El rendimiento es el resultado del efecto combinado de muchos factores tanto genéticos, como ecológicos (plagas y enfermedades), así como la interacción del genotipo con el medio ambiente En este estudio también se realizó una comparación de rendimientos, presentando pepino en espaldera el mayor rendimiento que pepino rastrero. También se realizó un análisis económico basado en un presupuesto parcial, a través del cual se pudo determinar que el tratamiento pepino en espaldera obtuvo mayor beneficio neto comparado con pepino rastrero que presentó un beneficio neto de US \$ 1736.44 dólares. González y Obregón, (2007) en chiltoma también realizaron un análisis económico basado en un presupuesto parcial, obteniendo el mayor beneficio neto el tratamiento microtúnel. Rodríguez y Morales, (2007) en tomate obtuvieron el mayor beneficio neto en el tratamiento microinvernadero. También En el 2009, Chavarría y Rizo en tomate obtuvieron el mayor beneficio neto en el tratamiento microinvernadero. Sevilla y Rodríguez también en el 2009 en chiltoma obtuvieron el mayor beneficio neto en el tratamiento Oberon seguido del tratamiento Vertimec.

\section{CONCLUSIONES}

El pepino sembrado en espaldera tiene un efecto sobre la ocurrencia poblacional de insectos plagas ya que se encontró un menor número de estos insectos comparado con pepino sembrado rastrero.

El sistema de siembra pepino rastrero tiene un efecto sobre la ocurrencia poblacional de insectos benéficos ya que encontró una tendencia de mayor número de estos insectos en pepino sembrado rastrero comparado con pepino sembrado en espaldera.

El sistema de siembra pepino en espaldera presentó el mejor rendimiento comparado con pepino sembrado rastrero. El sistema de siembra pepino en espaldera fue más rentable económicamente que el sistema de pepino rastrero ya que obtuvo mayor beneficio neto.

\section{RECOMENDACIONES}

Se sugiere a los pequeños y medianos productores del municipio de Tisma, el uso del sistema de siembra pepino en espaldera debido a que presentan mejores ventajas que el pepino sembrado rastrero, ya que se obtienen frutos de mejor calidad y mayores rendimientos.

Seguir realizando investigaciones sobre la técnica de siembra de pepino en espaldera, para generar mayor 
información, tomando en cuenta diferentes variables como ocurrencia poblacional de insectos plagas, benéficos, enfermedades y rendimiento, en diferentes zonas del país.

Se recomienda repetir este estudio en las zonas donde se siembra pepino para generar mayor información sobre el efecto que tienen el uso de estas dos técnica de siembra sobre la ocurrencia poblacional de insectos plagas, benéficos y su rendimiento.

\section{AGRADECIMIENTO}

Agradecemos al pueblo y Gobierno de Suecia por el apoyo económico brindado a los investigadores de la UNA a través de los fondos PACI y al productor de Tisma Francisco Altamirano.

\section{REFERENCIAS BIBLIOGRÁFICAS}

Altieri, M. A; Luigui, P.; Nicholls, C. 2007. El aporte de la naturaleza agricultura sostenible y procesos ecológicos; LEISA Revista de agroecología; v 22; $n^{\circ} 4$. 9p.

Amador, F; Tijerino, N. 2005. Insectos y Enfermedades Asociados al cultivo de Mora (Rubus glaucus, Benth), en la Sabana, Madriz. Tesis Ing. ISPAF. Managua, NI. UNA. 63 P.

AMUNIC. (Asociación de Municipios de Nicaragua). 2005. (Caracterización de municipios de Masaya). 2005. (en línea). Managua, Nicaragua Consultado el 7 de febrero del 2007. Disponible en http://www.pinoleros. com.

Argüello, H; Lastres, L.; Rueda, A. 2007a. Manual MIP en Cucúrbitas, Escuela Agrícola panamericana. Zamorano, Honduras. P 243.

Argüello, H; Lastres, L.; Rueda, A.; Rivera, M. 2007b. Guía para el reconocimiento y manejo de virosis en cultivos hortícolas. Programa de Manejo Integrado de Plagas en América Central (PROMIPAC-ZAMORANOCOSUDE). Carrera de Ciencia y Producción Agropecuaria. Escuela Agrícola Panamericana, Zamorano, Honduras. Pág. 97

Avelarez, j. 2001. Cultivo del pepino olericultura. Managua, UNA. P 12.

Bellman, H.; Senden, G. M. 1994. Arácnidos, Crustáceos y miriápodos; Guías de naturaleza Blume. trad, G. Castellá 1 ed. ES. Gayban. 36p.

Castillo, A.1970. Pruebas de fertilizante en el pepino .Rivas, Nicaragua. P 44.

Cáceres, E. 1980. Producción de hortalizas. Instituto interamericano de Ciencias Agrícolas San José, Costa Rica. P 387.

CENTA (Centro Nacional de Tecnología Agropecuaria y Forestal). 2003. Guía técnica cultivo del pepino, El salvador. p 16- 18 .

Chavarría, A.; Rizo, A. 2009. Evaluación de cinco alternativas de protección físicas y químicas de semilleros de tomate (Lycopersicum esculentum, Mill) contra el ataque del complejo mosca blanca (Bemisia tabaci Gennadius)- Geminivirus, en Tisma, Masaya. Tesis. Ing. ISPAF. Managua, NI. UNA. 45p.

Garache, M.; López, G. 2007. Efectos de policultivo Tomate (Lycopersicum esculentum, Mill), Chiltoma (Capsicum annum L.) y Maiz (Zea mays L.) en la ocurrencia poblacional de insecto plagas y artrópodos benéficos y el uso equivalente de la tierra Tisma - Masaya. Tesis Ing. ISPAF. Managua, NI, UNA. 75 P.

García, K.; Angulo, L. 2008. Efecto de cultivos en asocio Pepino (Cucumis sativus L.), Pipian (Cucúrbita pepo L.) y Fríjol de Vara (Vigna unguiculata L. Walp), en la ocurrencia poblacional de insectos plagas, benéficos y el rendimiento en Tisma, Masaya. Tesis Ing. ISPAF. Managua, NI, UNA. 87 p.

Gómez, J. 2006. Descripción del Comportamiento de Insectos y Enfermedades Asociadas al cultivo de Fresa (Fragaria spp, L) en el Municipio de la Sabana, Departamento de Madriz. Tesis Ing. Agrónomo. Managua, NI. UNA. p 114.

González, L. 2004. Alternativas de Manejo Integrado de Plagas con Insecticidas Biológicos y Botánicos en diferentes asocios de cultivos en la Comunidad de Pacora, San Francisco Libre. Tesis. Ing. ISPAF. Managua, NI, UNA. 59 P. 
González, J.; Obregón, H. 2007. Evaluación de alternativas de protección física y química de semilleros de chiltoma (Capsicum annum L.) contra el ataque de mosca blanca. (Bemisia tabaci, Gennadius) Geminivirus. Tesis Ing. ISPAF. Managua, NI, UNA. 73 p.

Hilje, L. 1993. Un esquema conceptual para el manejo integrado de la mosca blanca (Bemisia tabaci) en el cultivo del tomate. Manejo Integrado de Plagas, Turrialba, (Costa Rica) 29:51-57.

Huerres, C; Carballo, N.1998. Horticultura. Editorial, Pueblo y Educación P 70-72.

INEC. (Instituto Nacional de Estadísticas y Censo de Nicaragua) 1998. (en línea). http://www.inec.gob.ni/eva/ ende.htm.

INIFOM. (Instituto Nicaragüense de Fomento Municipal.). 2006. (en línea) Managua, Nicaragua. Consultado el 29 Abr 2008. Disponible en http://www.inifom.gob.ni/

Jiménez- Martínez E. 2008. Texto básico: Manejo Integrado de Plagas. Universidad Nacional Agraria UNAManagua, Nicaragua. 108 p.

MIDINRA. (Ministerio de desarrollo Agropecuario y reforma Agraria) 1984. Infamaciones agropecuarias. Boletín N $\mathrm{N}^{\circ}$. P 217.

Montes, A. 2005. Cultivo de hortalizas en el trópico, Escuela Agrícola panamericana. Departamento de Hortícola. Zamorano, Honduras. P 208.

Nicolls, C. y Altieri, M. A 1999. Manual práctico de control biológico para una agricultura sustentable. California, EUA. 7- 8 p.

Ospina. J. \& Alonso. H. 1998. Producción Agrícola, Enciclopedia Agrícola 2, Universidad Nacional de Colombia. P 552.

Pérez, G. D.; Sánchez, P. D. E. 2006. Efecto de policultivos (Tomate: Lycopersicum esculentum Mill) Pipián: Cucúrbita pepo L, Fríjol (Phaseolus vulgaris L.), en la incidencia poblacional de insectos plagas e insectos benéficos. Tesis Ing. Agr. Managua, NI, UNA.58p.

Rosset, R. P.1998. Aprovechamiento de la ecología y el comportamiento de los insectos mediante las técnicas de control cultural en el manejo de plagas.

Rodríguez, V.; Morales, J. 2007. Evaluación de alternativas de protección física y química de semilleros de tomate (Lycopersicum esculentum, Mill). Contra el ataque del complejo mosca blanca (Bemisia tabaci, Gennadius) Geminivirus y su efecto en el rendimiento, en el municipio de Tisma, Masaya. Tesis Ing. ISPAF. Managua, NI, UNA. 74p.

Sevilla, E.; Rodríguez, E. 2009. Evaluación de alternativas químicas y botánicas para el manejo del ácaro blanco (Poliphagotarsonemus latus, Bank.) en chiltoma (Capsicum annum L.). Tesis. Ing. ISPAF. Managua, NI. UNA. 40p. 